\title{
Author Correction: Cryo-EM structures of the full-length human KCC2 and KCC3 cation-chloride cotransporters
}

Ximin Chi iD, Xiaorong Li, Yun Chen, Yuanyuan Zhang, Qiang Su and Qiang Zhou

(c) CEMCS, CAS 2021

Cell Research (2021) 31:941; https://doi.org/10.1038/s41422-021-00536-3

Correction to: Cell Research https://doi.org/10.1038/s41422-02000437-x, published online 16 November 2020

The PDB and EMDB ID are lacked in the orignially published paper. The PDB IDs for KCC2, KCC3, and KCC3 bound DIOA are 6M23,
$6 \mathrm{M} 1 \mathrm{Y}$, and $6 \mathrm{M} 22$, respectively. The EMDB IDs for the corresponding cryo-EM maps are 30061, 30049, 30058, respectively. This correction does not affect the description and conclusion of this paper. 